\title{
Trends in the use of gluten-free claims on Canadian food labels between 2013-2017 and assessment of their nutritional quality
}

\section{Abstract}

Gluten-free claims (GFC) on food labels are becoming popular despite the fact that $1 \%$ and $6 \%$ of the population have celiac disorder or gluten sensitivity, respectively. A previous Canadian study found that GFC were displayed on $8 \%$ of food labels; however, certain food categories (e.g., snacks, meat products) were among the ones carrying most GFC. This study aimed to assess trends in the use of GFC on Canadian food labels in top food categories carrying GFC from 2013 to 2017 and to determine the nutritional quality between products with and without GFC.

This study was a repeated cross-sectional analysis of the University of Toronto Food Label Information Program 2013-2017. GFC were identified by systematically reviewing the photographs of food labels $(n=9,179)$ in seven food categories previously identified as carrying larger proportions of GFC: 1) desserts; 2) desserts toppings and fillings; 3) meat products; 4) nuts and seeds; 5) sauces and dips; 6) snacks; and 7) soups. GFC were coded as present, if a gluten-free declaration or symbol was made on package, otherwise products were coded as claim absent. Proportions of products displaying GFC were calculated overall and by food category. Mean contents of saturated fat $(\mathrm{g}$ per $100 \mathrm{~g} / \mathrm{ml})$, sodium $(\mathrm{mg}$ per $100 \mathrm{~g} / \mathrm{ml})$ and sugar $(\mathrm{g}$ per $100 \mathrm{~g} / \mathrm{ml})$ were calculated for products with and without GFC. Chi-square and Mann-Whitney-Wilcoxon tested differences in proportions and mean contents of those nutrients between years.

Results showed that among these categories, GFC have significantly increased from 11\% in 2013 to $23 \%$ in 2017 (p $<0.001$ ). At a category level, snacks had the greatest increase of GFC as their prevalence doubled $(15 \%$ to $33 \%, \mathrm{p}<0.001)$, followed by nuts and seeds $(12 \%$ to $27 \%, \mathrm{p}<0.001)$ and meat products $(15 \%$ to $28 \%, \mathrm{p}<0.001)$, respectively for 2013 and 2017 . The proportion of GFC in dessert toppings and fillings remained constant ( $16 \%$ in 2013 and $14 \%$ in 2017, p $=0.74)$. When the nutritional composition was examined, results were mixed: in dessert toppings and filling, meat products and, nuts and seeds, products with GFC had higher contents of saturated fat, sodium and sugar in comparison to their counterpart without claims, whereas the opposite was true for foods within desserts, sauces and dips, snacks, and soups categories $(\mathrm{p}<0.001$ for all nutrients, except for saturated fat in nuts and seeds). In conclusion, prevalence of GFC has doubled in the Canadian food supply; such claims are not indicative of better nutritional composition.

\section{Conflict of Interest}

Prior coming to the University of Toronto, Beatriz Franco-Arellano was a PepsiCo Mexico employee. The company had no connection or funding to the research. Mary L'Abbé declares that she has received research grants from the Canadian Institutes of Health Research, Canadian Stroke Network, Burroughs Wellcome Fund, Heart and Stroke Foundation of Canada, International Development Research Centre, University of Toronto. 